\title{
Cold-inducible RNA binding protein promotes breast cancer cell malignancy by regulating Cystatin $\mathrm{C}$ levels
}

\author{
ALBERTO INDACOCHEA, ${ }^{1,2}$ SANTIAGO GUERRERO, ${ }^{1,5}$ MACARENA UREÑA, ${ }^{2}$ FERRÁN ARAUJO, ${ }^{2}$ \\ OLGA COLL, ${ }^{1}$ MATILDE E. LLEONART, ${ }^{2,3}$ and FÁTIMA GEBAUER ${ }^{1,4}$ \\ ${ }^{1}$ Gene Regulation, Stem Cells and Cancer Programme, Centre for Genomic Regulation (CRG), The Barcelona Institute of Science \\ and Technology, 08003 Barcelona, Spain \\ ${ }^{2}$ Biomedical Research in Cancer Stem Cells, Vall d'Hebron Research Institute (VHIR), 08035 Barcelona, Spain \\ ${ }^{3}$ Spanish Biomedical Research Network Centre in Oncology, CIBERONC, Spain \\ ${ }^{4}$ Universitat Pompeu Fabra (UPF), 08003 Barcelona, Spain
}

\begin{abstract}
Cold-inducible RNA binding protein (CIRBP) is a stress-responsive protein that promotes cancer development and inflammation. Critical to most CIRBP functions is its capacity to bind and posttranscriptionally modulate mRNA. However, a transcriptome-wide analysis of CIRBP mRNA targets in cancer has not yet been performed. Here, we use an ex vivo breast cancer model to identify CIRBP targets and mechanisms. We find that CIRBP transcript levels correlate with breast cancer subtype and are an indicator of luminal A/B prognosis. Accordingly, overexpression of CIRBP in nontumoral MCF-10A cells promotes cell growth and clonogenicity, while depletion of CIRBP from luminal A MCF-7 cells has opposite effects. We use RNA immunoprecipitation followed by high-throughput sequencing (RIP-seq) to identify a set of 204 high confident CIRBP targets in MCF-7 cells. About $10 \%$ of these showed complementary changes after CIRBP manipulation in MCF-10A and MCF-7 cells, and were highly interconnected with known breast cancer genes. To test the potential of CIRBP-mediated regulation of these targets in breast cancer development, we focused on Cystatin C (CST3), one of the most highly interconnected genes, encoding a protein that displays tumor suppressive capacities. CST3 depletion restored the effects of CIRBP depletion in MCF-7 cells, indicating that CIRBP functions, at least in part, by down-regulating CST3 levels. Our data provide a resource of CIRBP targets in breast cancer, and identify CST3 as a novel downstream mediator of CIRBP function.
\end{abstract}

Keywords: CIRBP; CST3; breast cancer

\section{INTRODUCTION}

RNA binding proteins orchestrate posttranscriptional control of gene expression and are emerging as important modulators of cancer progression (Wurth and Gebauer 2015; Pereira et al. 2017; Moore et al. 2018; GarcíaCárdenas et al. 2019). Cold-inducible RNA binding protein (CIRBP, also termed CIRP and hnRNP A18) is a stress-responsive protein that partially relocates from the nucleus to the cytoplasm under diverse types of stress such as mild hypothermia, UV-irradiation, endoplasmic reticulum (ER) stress or hypoxia (Lujan et al. 2018). In the cytoplasm, CIRBP binds to the coding sequence and $3^{\prime}$-UTRs of target transcripts and promotes or stabilizes mRNA levels. For ex-

${ }^{5}$ Present address: Centro de Investigación Genética y Genómica, Facultad de Ciencias de la Salud Eugenio Espejo, Universidad UTE, 170129 Quito, Ecuador

Corresponding authors: fatima.gebauer@crg.eu, matilde.lleonart@vhir.org, santiago.guerrero@ute.edu.ec

Article is online at http://www.rnajournal.org/cgi/doi/10.1261/rna. 076422.120. Freely available online through the RNA Open Access option. ample, CIRBP promotes telomere maintenance by increasing the levels of TERT mRNA (Zhang et al. 2016), and enhances the tumorigenic properties of cancer cells by promoting the stability of HIF1 $\alpha$ and cyclin $E$ mRNAs (Guo et al. 2010; Chang et al. 2016). However, other modes of CIRBP-mediated regulation have also been reported. For instance, CIRBP stimulates global protein synthesis by promoting 4E-BP1 phosphorylation (ArteroCastro et al. 2009; Chang et al. 2016), and has been proposed to stimulate mRNA-specific translation (Yang et al. 2006; Chang et al. 2016), although direct evidence for the latter is still missing. In addition, CIRBP regulates circadian gene expression by promoting alternative polyadenylation (APA) of target transcripts (Morf et al. 2012; Liu et al. 2013). Under UV-irradiation, CIRBP is transiently recruited to sites of DNA damage, where it promotes repair by modulating the association of DNA repair complexes (MRN and

(C) 2021 Indacochea et al. This article, published in RNA, is available under a Creative Commons License (Attribution 4.0 International), as described at http://creativecommons.org/licenses/by/4.0/. 
ATM kinase) to DNA damage sites (Chen et al. 2018). CIRBP also stimulates the ERK pathway, an event important for bypass of replicative senescence and protection from apoptosis (Sakurai et al. 2006; Artero-Castro et al. 2009). Intriguingly, CIRBP can augment inflammation and induce pyroptosis by a mechanism presumably unrelated to its RNA-binding capacity. Upon hemorrhagic shock and sepsis, CIRBP is released into the circulation where it binds to the TLR4-MD2 receptor of macrophages, leading to release of TNF $\alpha$, inflammation and tissue injury (Qiang et al. 2013).

Most of the functions mentioned above are consistent with a pro-oncogenic role of CIRBP. Accordingly, although CIRBP null mice display no obvious phenotype under normal laboratory conditions, they show attenuated hepatocarcinogenesis upon thioacetamide treatment and are less susceptible to dextran sodium sulfate-induced intestinal inflammation and tumorigenesis (Sakurai et al. 2006, 2014, 2015; Masuda et al. 2012).

Despite the relevance of CIRBP in tumor progression, no transcriptome-wide attempts have been done to identify CIRBP targets in a cancerous context. Here we use RNA immunoprecipitation followed by high-throughput sequencing (RIP-seq) to identify CIRBP targets. We focus on breast cancer because (i) previous in vitro evidence indicated that CIRBP could modulate the tumoral properties of MDA-MB-231 breast cancer cells (Chang et al. 2016), (ii) immunohistochemistry $(\mathrm{IHC})$ analysis pointed to the presence of CIRBP in the cytoplasm of cells from breast cancer patients (Artero-Castro et al. 2009), and (iii) the large number of samples present in databases compared to other tumor types allows for higher statistical relevance. We first validated breast cancer as a suitable model to study CIRBP-mediated regulation. Indeed, analysis of patient samples indicated that CIRBP mRNA levels correlate with breast tumor subtype and are an indicator of prognosis for the luminal A/B subtype. Depletion of CIRBP from luminal A MCF-7 breast ductal carcinoma cells resulted in decreased proliferation and clonogenicity while, conversely, CIRBP overexpression in nontumoral breast MCF-10A cells increased these traits. RIP-seq from MCF7 cells identified 204 high-confident targets, $80 \%$ of them novel. To identify functionally relevant targets, we used the MCF-7/MCF-10A cell pair and compared transcriptome changes after CIRBP depletion or overexpression, respectively. Of the 204 targets, 22 showed consistent changes in both cell lines. Network analysis indicated that 13 of these targets were highly interconnected with known breast cancer genes. We provide proof-of-principle analysis showing that one of these novel targets, Cystatin C (CST3) is a functionally relevant CIRBP target in breast cancer, as depletion of CST3 abrogates the deleterious effects of CIRBP depletion. These data indicate that CST3 is a novel mediator of CIRBP function in breast cancer.

\section{RESULTS}

\section{CIRBP is up-regulated in luminal breast cancer and its expression correlates with poor clinical outcome}

To assess the relevance of CIRBP in cancer, we first interrogated CIRBP expression in patient databases using cBioPortal (https://www.cbioportal.org/) (Cerami et al. 2012; Gao et al. 2013). TCGA database analysis indicated that CIRBP is altered in a variety of human tumors, the most frequent event consisting of mRNA up-regulation (Fig. 1A). We next focused on breast cancer, which had the highest absolute number of CIRBP alterations. Breast cancer is classified in four main subtypes based on the expression of molecular markers, namely estrogen receptor (ER), progesterone receptor (PR) and human epithelial growth factor receptor 2 (HER2) (Harbeck et al. 2019). In increasing order of aggressiveness, tumors are low proliferative $\mathrm{ER}^{+} \mathrm{PR}^{+} \mathrm{HER} 2^{-}$(luminal $\mathrm{A}$ ), high proliferative $\mathrm{ER}^{+} \mathrm{PR}^{+}$ HER2 ${ }^{-}$(luminal B), HER2 ${ }^{+}$(luminal B and nonluminal) or $\mathrm{ER}^{-} \mathrm{PR}^{-} \mathrm{HER2} 2^{-}$(triple negative). Analysis of CIRBP expression along these subtypes revealed a higher transcript expression in less aggressive hormone-positive $\left(E R^{+} P R^{+}\right)$ breast cancer samples present in TCGA and METABRIC databases (Fig. 1B). Although less aggressive, hormone-positive breast cancer represents the most abundant class (60\%-70\%), and can metastasize leading to death. To test whether CIRBP levels can function as a prognosis factor, we explored CIRBP transcript prevalence in $\mathrm{ER}^{+} \mathrm{PR}^{+}$patients with primary infiltrating ductal carcinoma and negative lymph node status. The results indicated that CIRBP up-regulation correlates with poor disease-free survival (Fig. 1C).

In order to select an appropriate cell system to study the function and targets of CIRBP in breast cancer, we next explored CIRBP protein levels in a set of breast cancer cell lines. CIRBP was overexpressed in luminal (MCF-7, T47D, BT474) compared with nonluminal (MDA-MB-468 and SKBR3) and nontumoral (MCF-10A) cells (Fig. 1D). We, thus, selected the luminal MCF-7 and the nontumoral MCF-10A cell line pair for our study.

\section{CIRBP promotes proliferation}

To evaluate the role of CIRBP in our cellular model, we both overexpressed CIRBP in MCF-10A cells and downregulated CIRBP in MCF-7 cells. Even mild overexpression of CIRBP (to $~ 30 \%$ of endogenous CIRBP levels) resulted in increased cell proliferation and clonogenicity (Fig. 2A-C), while depletion of CIRBP had the opposite effect (Fig. 2D-F). These results are consistent with a pro-oncogenic role of CIRBP in breast cancer.

\section{Transcriptome-wide identification of CIRBP targets}

To identify CIRBP mRNA targets in MCF-7 cells in an unbiased, transcriptome-wide manner, we used RNA 

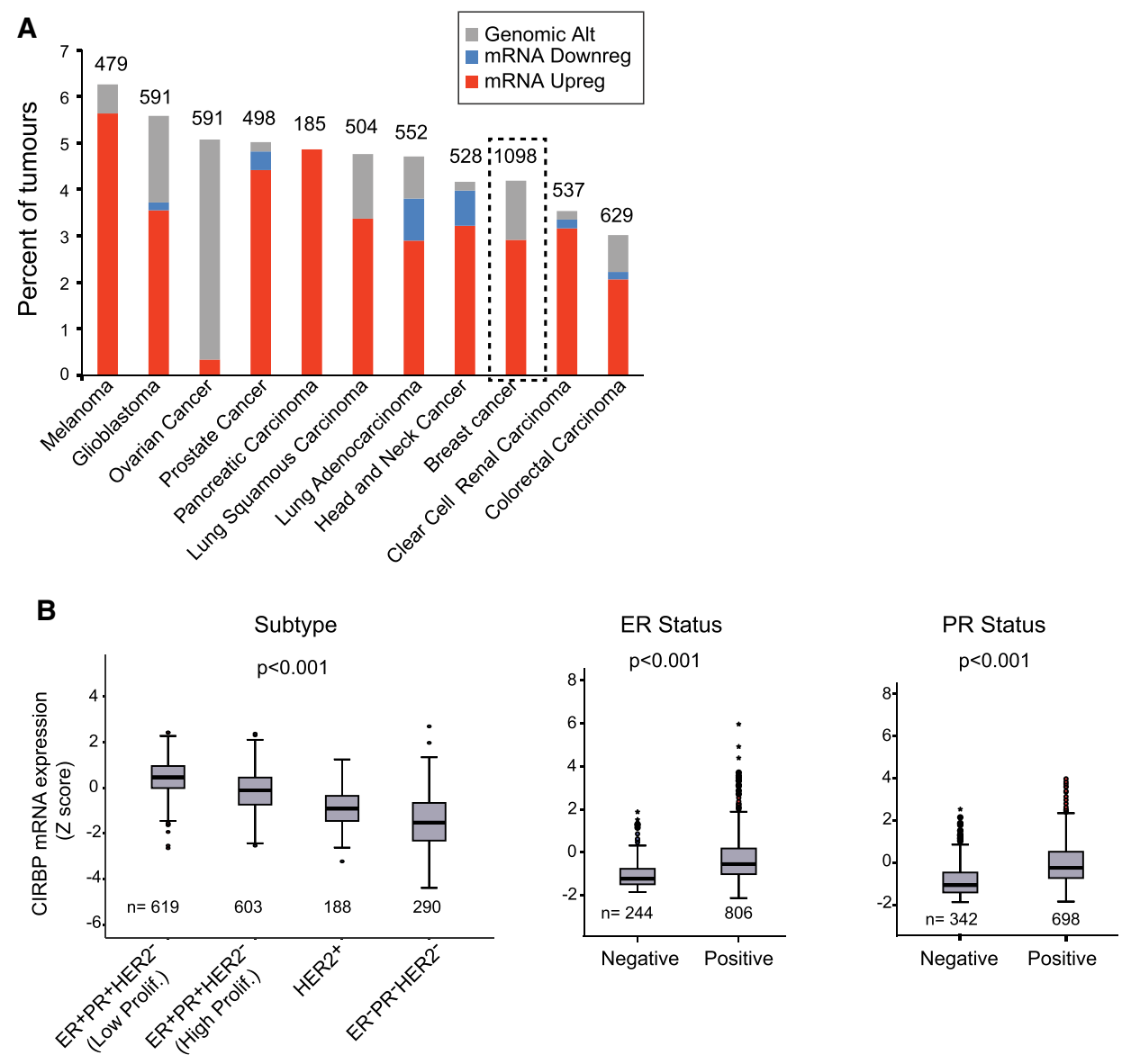

C

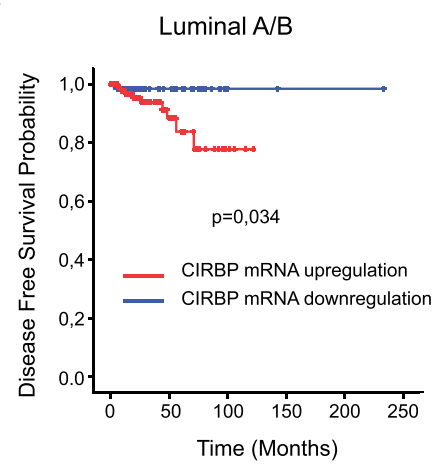

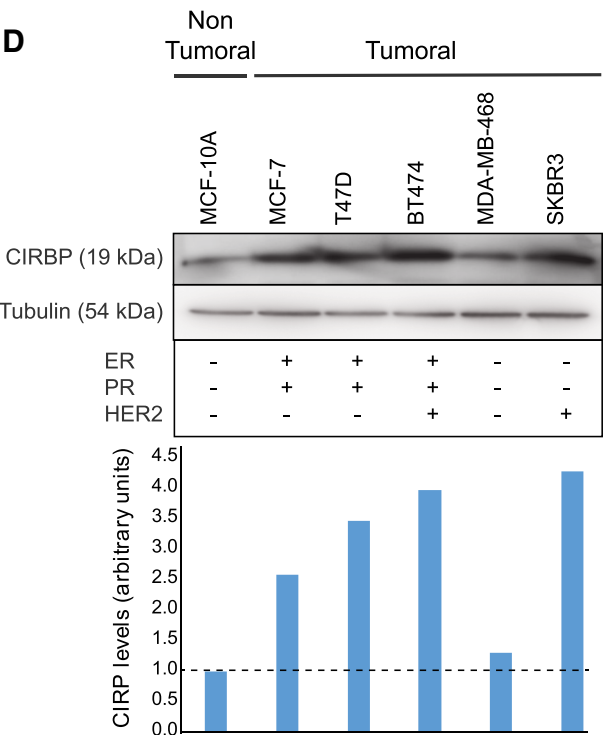

FIGURE 1. CIRBP is up-regulated in luminal breast cancer and correlates with poor clinical outcome. (A) CIRBP genomic alterations (mutations, deletions, and amplifications) and mRNA transcript levels were evaluated across cancer types using the TCGA database. (B) Expression of CIRBP mRNA across breast cancer subtypes using METABRIC (left panel) and TCGA (middle and right panels) databases. Box-plot center line represents the median, box limits indicate the 25th and 75th percentiles, whiskers extend 1.5 times the interquartile range, and dots represent outliers. Statistics were performed using Kruskal-Wallis (left panel) and Mann-Whitney U (middle and right panels). (C) Kaplan-Meier curve for disease free survival in patients with infiltrating ductal carcinoma of the luminal A/B subtype, and negative lymph node. The optimal cut point was found using the Cut-off Finder script for R. Differences between groups was calculated using log rank test. (D) Western blot analysis of CIRBP levels in breast cancer cell lines. Tubulin is shown as loading control. The molecular markers of these lines are indicated in the chart below. Quantification of the CIRBP signal corrected for tubulin is shown at the bottom. 
A

MCF-10A/ CIRBP OE

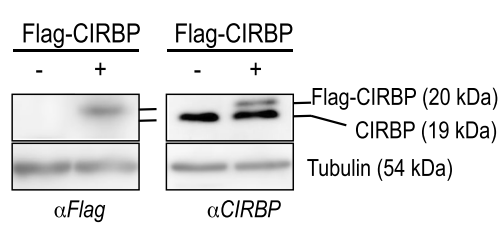

B

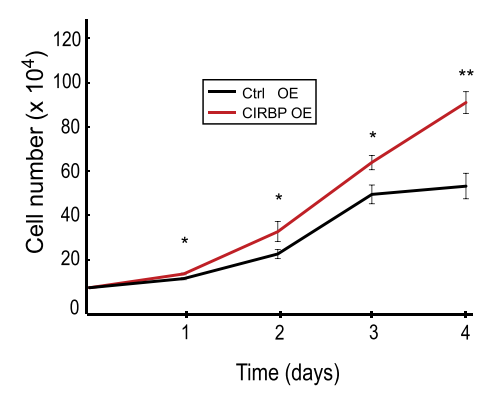

C
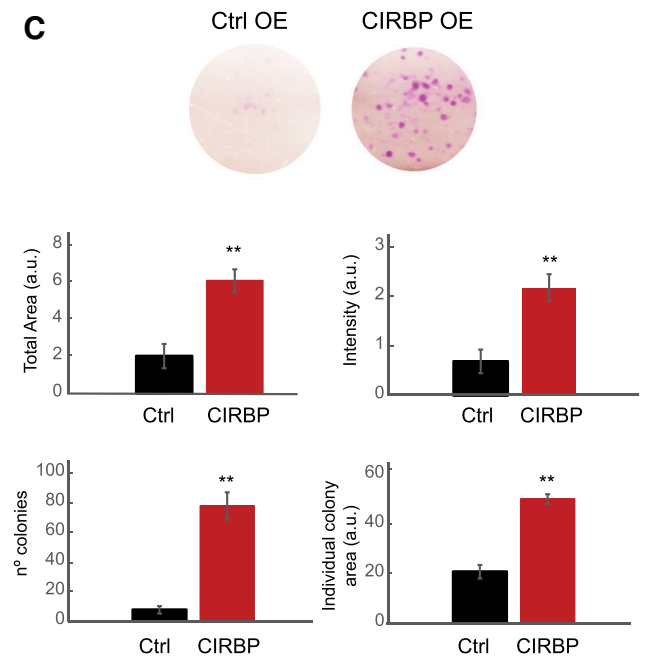

D

MCF-7/ siCIRBP

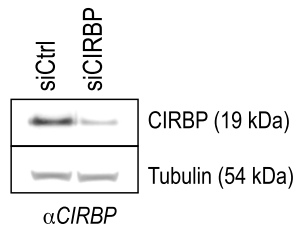

E

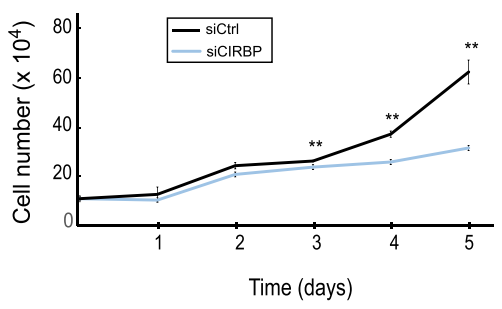

F
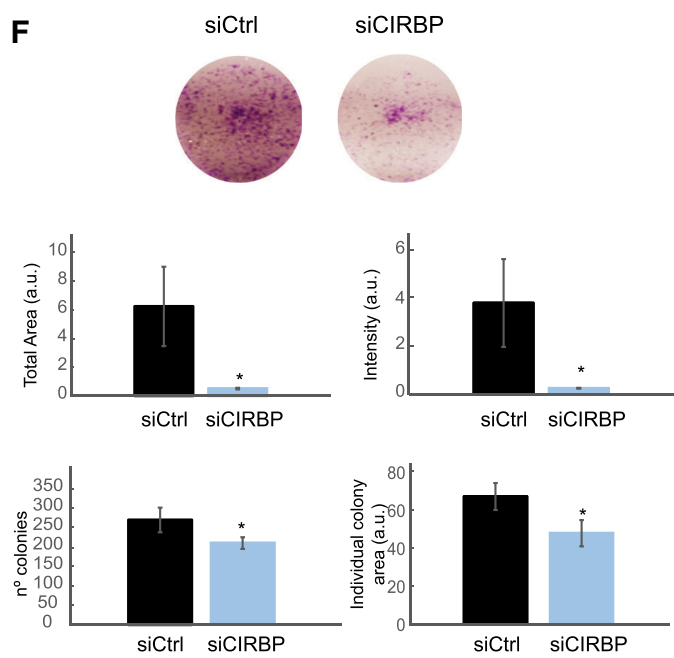

FIGURE 2. CIRBP promotes tumorigenic traits. (A) MCF-10A cells were stably transduced with a retroviral vector expressing CIRBP (+), or with the empty vector (-) as control. Western blots with anti-Flag (left) or anti-CIRBP (right) antibodies are shown. These cells were tested for proliferation $(B)$ and clonogenicity $(C)$. (D) MCF-7 cells were transiently transfected with siRNA pools against CIRBP or with a control siRNA pool. The efficiency of depletion was assessed by western blot at the beginning of the experiment (day 0$)$. These cells were tested for proliferation (E) and clonogenicity $(F)$. Error bars represent the standard deviation of triplicate experiments. Statistical significance was assessed by Student's $t$-test $\left(*^{*}\right) P<0.05$, (**) $P<0.01$

immunoprecipitation followed by high-throughput sequencing (RIP-seq) (Fig. 3A). Given that CIRBP is located in the nucleus of MCF-7 cells, we chose a cell lysis protocol that included sonication but only mild detergents to preserve RNA-protein interactions. CIRBP was immunoprecipitated from the lysates and the associated RNA sequenced using poly(A) RNA-seq. Parallel reactions with nonspecific rabbit lgG were carried as negative control. The IP efficiency of duplicate experiments is shown in Figure 3B. As expected, only a small proportion of the input RNA was associated to CIRBP (Supplemental Fig. S1A) and the obtained sequences are biased toward the mRNA $3^{\prime}$ end (Supplemental Fig. S1B). We believe that this bias reflects the chosen protocol for the identification of targets rather than an intrinsic property of CIRBP binding, because sonication shears the RNA and poly(A) selection then enriches for 3 ' fragments. However, we cannot rule out a preferential binding of CIRBP to $3^{\prime}$-UTRs in MCF-7 cells, as reported in other contexts (Morf et al. 2012; Liu et al. 2013). Sequencing of the two RIP replicates showed a high correlation (Supplemental Fig. S1C). We performed pairwise comparisons of CIRBP IP versus IgG or CIRBP IP versus input, considering either all counts for each gene or only counts in the last three exons to account for the 
A

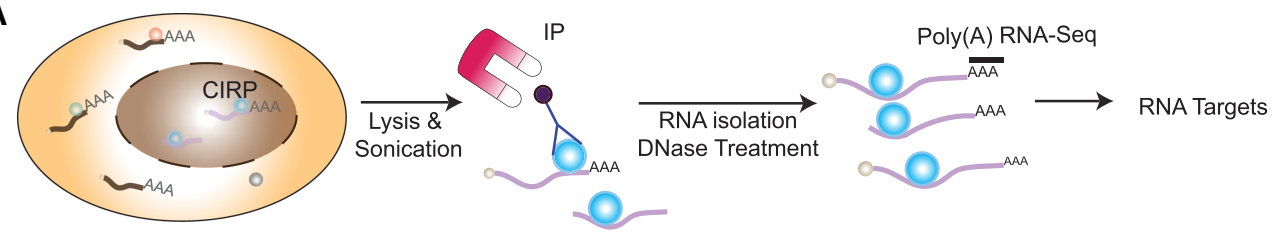

B

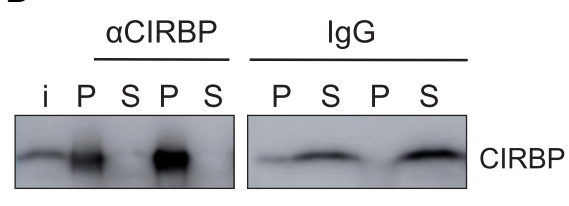

C

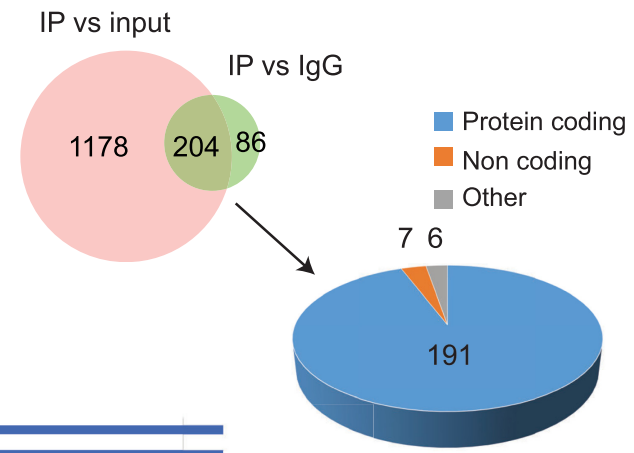

D

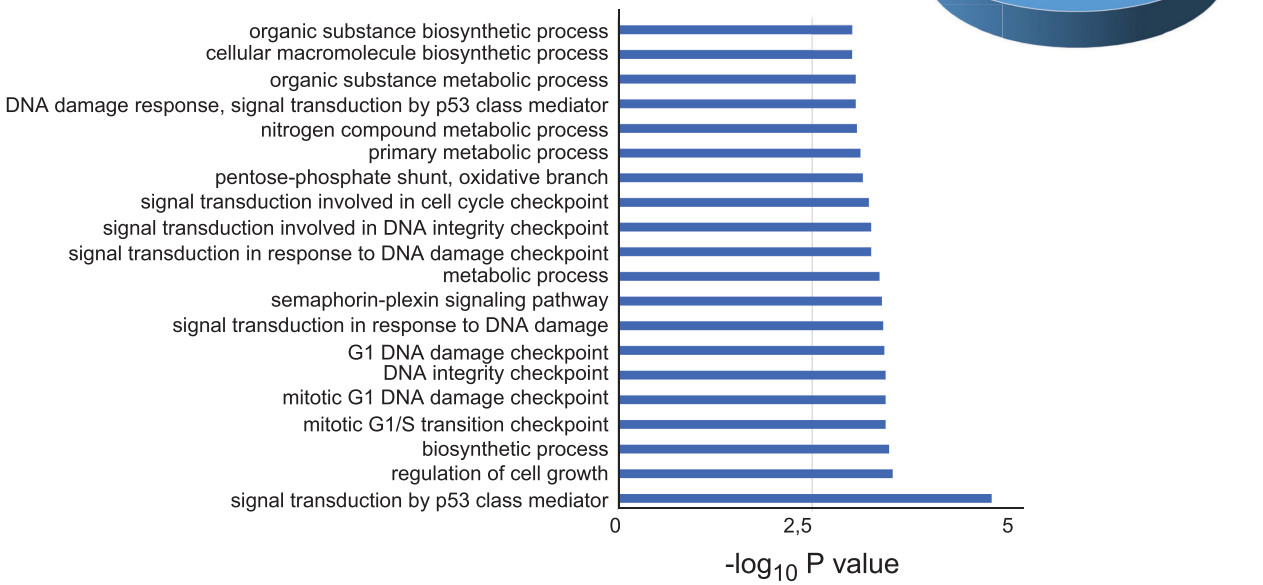

E

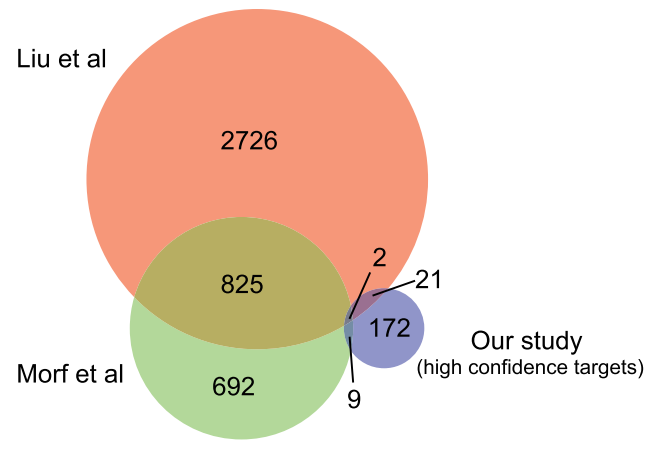

FIGURE 3. Identification of CIRBP targets. (A) Schematic representation of our RIP-seq approach to identify CIRBP targets. (B) Efficiency of immunoprecipitation in duplicate experiments. Immunoprecipitations with nonspecific lg $\mathrm{G}$ were carried in parallel as control. (i) input, (P) pellet, (S) supernatant. (C) A target was considered positive when there was significant enrichment ( $\left.22 F C>1, P_{a d j}<0.01\right)$ in the CIRBP IP versus the input, or in the CIRBP IP versus the IgG control. Targets positive in both analysis (204) were considered high-confident targets. Most of these targets are protein-coding genes (right). (D) Gene Ontology (GO) analysis of the high confidence targets. The top 20 biological processes were selected for representation. (E) Comparison between CIRBP high-confidence targets identified in this study and previous transcriptome-wide analysis of CIRBP targets.

3' bias (Supplemental Fig. S1D; Supplemental Table S1). A larger number of targets were retrieved in the CIRBP versus input comparison, while minimal differences were observed using whole gene or last exons counts (Fig. 3C;
Supplemental Fig. S1D). Independent RT-qPCR validation of targets selected over a wide range of $P$-values yielded a validation rate of $73 \%$ (Supplemental Fig. S1E). We reasoned that robust CIRBP targets should be enriched over 
both the input and the lgG control; thereby we selected a subset of 204 targets enriched under both comparisons as our high-confident target list (Fig. 3C; Supplemental Table S1). Around $90 \%$ of these targets are protein-coding genes while the remaining $10 \%$ include long noncoding RNAs and pseudogenes (Fig. 3C; Supplemental Table S1). Gene Ontology (GO) analysis revealed biological processes related with DNA damage and proliferation, in line with previous reports showing that CIRBP is involved in the DNA damage response (Fig. 3D; Yang and Carrier 2001; Yang et al. 2006, 2010; Chen et al. 2018). However, none of our targets had been described in previous CIRBP analyses, suggesting novel mediators for DNA damage regulation by CIRBP. We further compared our list with CIRBP targets defined in two previous transcriptome-wide efforts aimed at the study of circadian gene regulation in the mouse (Morf et al. 2012; Liu et al. 2013). The comparison revealed an overlap of 32 targets ( $15 \%$ of our list) (Fig. $3 \mathrm{E})$. This limited overlap might be due to the different organism (human vs. mice), cell types (epithelial cells vs. fibroblasts), biological contexts (cancer vs. nontumoral) and technologies (RIP vs. CLIP or PAR-CLIP) used in our study compared to previous reports. Altogether, the results indicate that we have identified novel targets of CIRBP in human luminal breast cancer cells.

\section{CIRBP regulates a network of breast cancer genes}

As mentioned above, CIRBP has been shown to posttranscriptionally regulate the levels of target transcripts. In order to identify functionally relevant CIRBP targets in breast cancer, we evaluated changes in the transcriptomes of MCF-10A and MCF-7 cells after overexpression and depletion of CIRBP, respectively, by RNA-seq analysis (Supplemental Table S2). Most changes observed for the 204 CIRBP targets included up-regulation upon CIRBP depletion in MCF-7 cells or down-regulation in MCF-10A cells upon CIRBP overexpression, indicating a predominant role of CIRBP in mRNA down-regulation (Fig. 4A, right panel). A set of 1613 transcripts changed in both cell types, of which 28 overlapped with our high-confident CIRBP target list (Fig. 4A, left panel; Supplemental Table S2). A close examination of the magnitude and direction of changes in these 28 targets indicated that 22 of them changed in opposite directions in both cell lines, consistent with variations of CIRBP levels (Fig. 4B).

To identify which of these 22 genes was potentially relevant in breast cancer, we performed network analysis. A list of known breast cancer genes was constructed from KEGG (https://www.genome.jp/kegg/), COSMIC (https ://cancer.sanger.ac.uk/cosmic) and TCGA databases. This list was intersected with the 22 CIRBP targets using Ingenuity Pathways allowing for direct and up to two indirect interactions. Strikingly, 13 of the 22 targets (59\%) were highly interconnected with known breast cancer genes:
CST3, S100A11, MAPK11, STK11, CDT1, HOMER3, TRIM28，RPL28, NCAPH2, SF3A2, SLC25A10, SEZ6L2, and MEIS3 (Fig. 4C). Of these, TRIM28 is a known breast cancer gene (Wei et al. 2016; Czerwińska et al. 2017), while the rest are novel. Of note, four of the cancer genes present in the network (MAP2K2, E2F1, FZD2, and WNT7B), although absent from our high-confident list of CIRBP targets, are enriched in CIRBP IPs (Supplemental Table S1). These data indicate that our stringent criteria may have resulted in loss of true positives and, together with the high percentage of CIRBP targets included in the network, provides confidence to our selection.

\section{CIRBP promotes oncogenic traits through regulation of CST3}

To validate CIRBP regulation of select targets, we focused on three of the highly interconnected genes: CST3, SLC25A10, and TRIM28. We corroborated the RNA-seq results obtained after CIRBP depletion and overexpression by using RT-qPCR (Fig. 5A). Results were further validated by western blot after depletion of CIRBP from MCF-7 cells (Fig. 5B).

As a proof of principle, we chose CST3 for functional studies. Our aim was to test whether CIRBP-mediated regulation of CST3 was relevant to modulate the tumorigenic properties of MCF-7 cells. To that end, and given that CIRBP down-regulates CST3 levels, we tested whether CST3 depletion rescues the phenotype resulting from CIRBP depletion. The results showed that, while CST3 silencing alone had minor effects on MCF-7 clonogenicity, it did overcome the reduced clonogenicity resulting from CIRBP depletion (Fig. 5C, see efficiency of depletion in Supplemental Fig. S2A). Similar results were obtained when MCF-7 cells were grown in nonadherent plates. In these conditions, MCF-7 cells grow as 3D-aggregates whose size and viability decrease after CIRBP knockdown. Concomitant depletion of CST3 eliminates and even surpasses this effect (Fig. 5D, see efficiency of depletion in Supplemental Fig. S2B). These results indicate that downregulation of CST3 mRNA levels by CIRBP contributes to the oncogenic features of MCF-7 breast cancer cells.

\section{DISCUSSION}

Despite the fact that luminal tumors $A$ and $B$ have a better prognosis (compared to Her2+ and TN), in the last decades an increase in the number of luminal $A / B$ patients that have recurred has been detected. Therefore, it is essential to identify new biomarkers capable of predicting which subgroup of patients may not respond adequately to therapy or recur. RNA binding proteins are gaining great attention in the cancer field for their capacity to modulate virtually all cancer hallmarks (Pereira et al. 2017). Although they constitute one of the largest protein families in the 
A

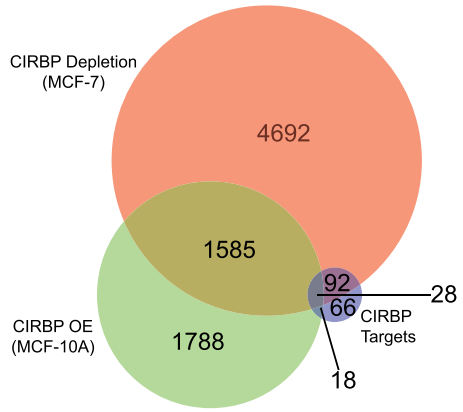

B

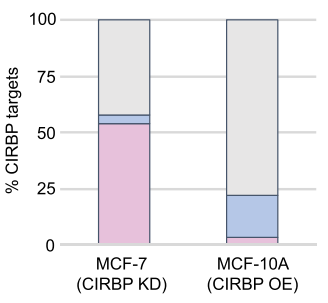

$\square$ Unchanged

$\square$ Downregulated

$\square$ Upregulated

CIRBP OE in MCF-10A CIRBP KD in MCF-7

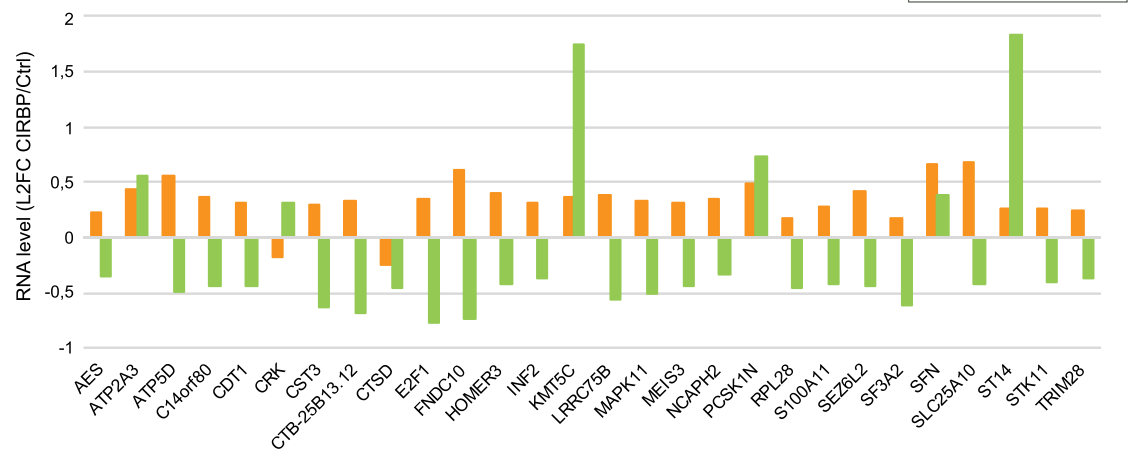

C

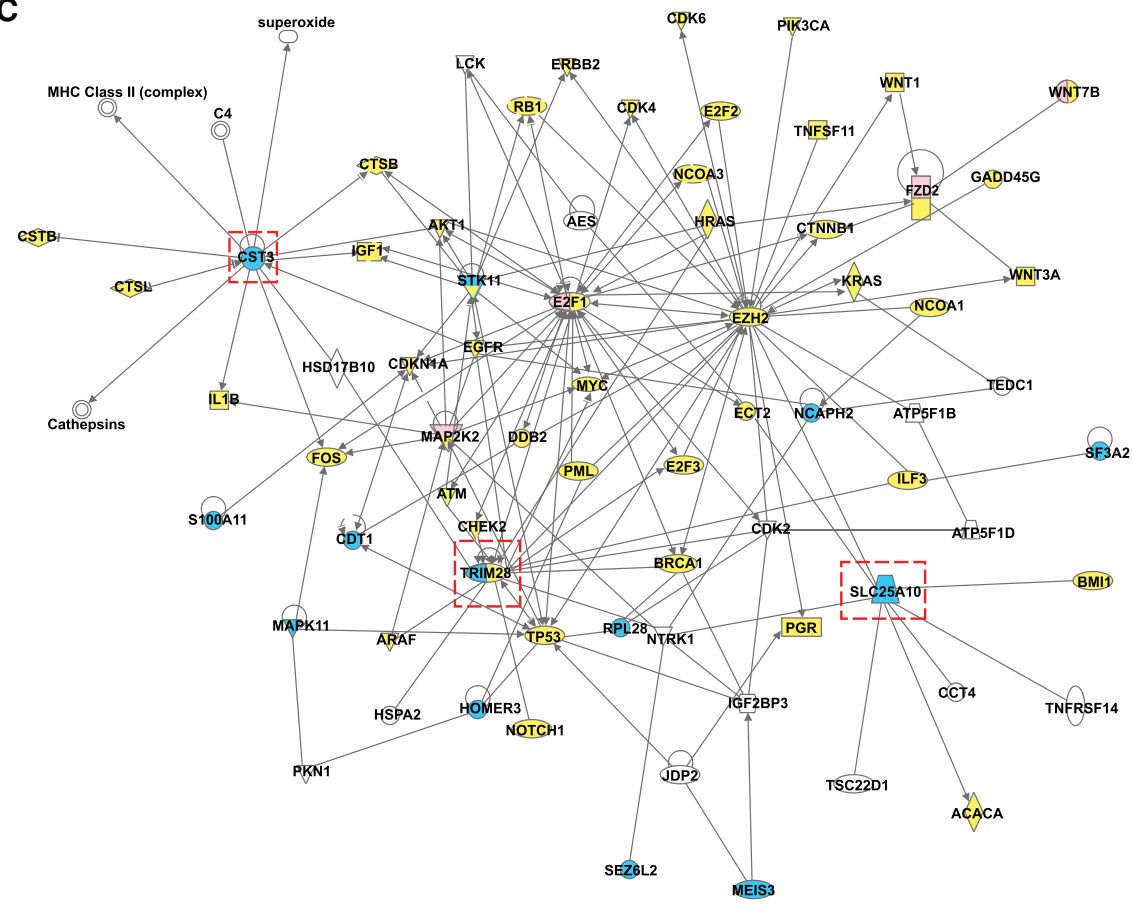

FIGURE 4. CIRBP regulates a network of breast cancer genes. (A) Differences in the transcriptomes of MCF-10A cells overexpressing CIRBP, and MCF-7 cells depleted of CIRBP, with their respective controls were determined by RNA-seq (experiments were performed in duplicate; threshold for significance, $\left.P_{\text {val }}<0.05\right)$. The overlap between genes changing in both cell lines and the CIRBP high-confident target list is shown on the left. The direction of change in the high-confident target list is shown on the right. (B) Detail of the direction and magnitude of change of the $28 \mathrm{CIRBP}$ targets overlapping in A. (C) Network analysis of CIRBP targets (see text for details). Yellow, known breast cancer gene; blue, CIRBP high-confident target; pink, CIRBP target identified in this study but excluded from the high-confident list. A few targets show two colors, indicative of their classification in two groups. Connections represent interactions at any reported level (i.e., protein-protein, protein-mRNA/DNA, activation); arrows represent activation while bars represent inhibition. Shapes of the nodes: vertical ellipse, transmembrane receptor; horizontal ellipse, transcription regulation; diamond, enzyme; square, cytokine; inverted triangle, kinase; vertical rectangle, G-protein coupled receptor; horizontal rectangle, ligand-dependent nuclear receptor; trapezoid, transporter; simple circle, other; concentric circle, group or complex. Dashed, red squares represent CIRBP targets that are highly interconnected in the network and which were chosen for independent validation. 
A

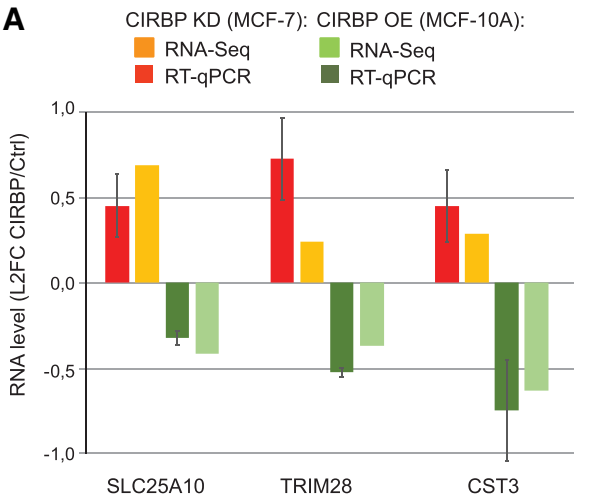

C

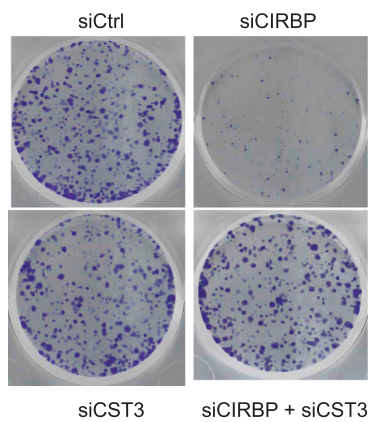

D

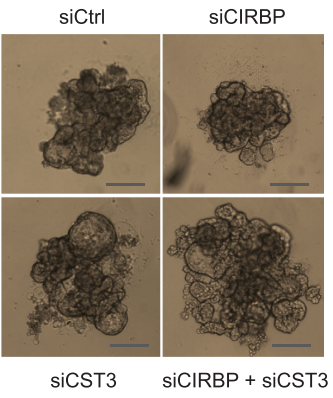

B
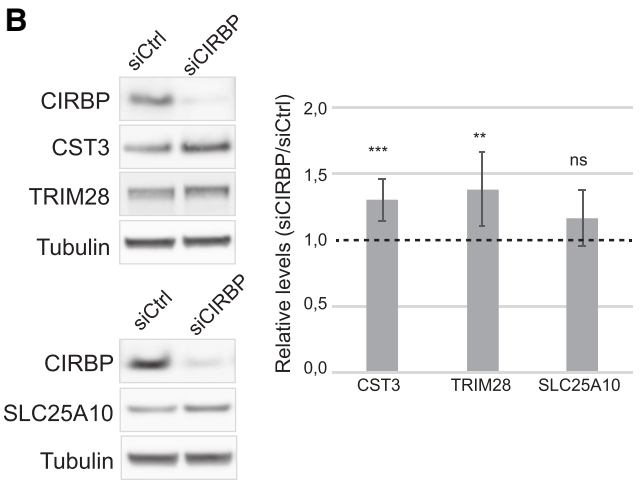

FIGURE 5. CST3 mediates CIRBP regulation of cancerous traits. (A) Validation of RNA-seq results by RT-qPCR of selected targets after manipulation of CIRBP levels in MCF-7 and MCF-10A cells. For comparison, the RNA-seq data are included. Error bars represent the standard deviation of at least two independent experiments. (B) Levels of targets in A by western blot after depletion of CIRB from MCF-7 cells. Tubulin was used as a loading control. Quantification of at least four experiments, corrected for tubulin and normalized to the siControl (dashed line) is shown on the right. (C) Clonogenic assay after depletion of CIRBP and/or CST3 from MCF-7 cells. A representative image is shown per condition on the left. Middle and right panels show the quantification of the area of colonies and global plate intensity, respectively. Error bars represent the standard deviation from triplicates carried in parallel. Two independent biological replicates were performed. (D) Anchorage independent growth of MCF7 cells after depletion of CIRBP and/or CST3. Representative images are shown on the left. Relative viability and size of aggregates are shown in the middle and right, respectively. Error bars represent standard deviation $(n=6)$. Significance was determined using Student's $t$-test. (***) $P_{\text {val }}<$ $0.001,\left({ }^{*}\right) P_{\text {val }}<0.01,\left({ }^{*}\right) P_{\text {val }}<0.05$; (ns) nonsignificant.

cell, understanding of their roles in disease is in its infancy (Castello et al. 2013; Gerstberger et al. 2014; Wurth and Gebauer 2015; Hentze et al. 2018). Here we show that the RNA binding protein CIRBP displays pro-oncogenic properties in breast cancer, consistent with previous reports (Chang et al. 2016). Importantly, CIRBP transcript levels correlate with poor prognosis in the most frequent luminal $A / B$ breast cancer subtype. To understand the role of CIRBP in luminal breast cancer, we perform the first genome-wide identification of CIRBP targets in cancer, and provide a repository of novel targets with potential relevance in breast oncogenesis. Among them, we identify CST3 as a functionally relevant target that is down-regulated by CIRBP to promote the tumorigenic properties of breast cancer cells.

The most significant $\mathrm{GO}$ terms associated with our list of CIRBP targets are related to the DNA damage response, in agreement with the reported function of CIRBP in this 
biological process (Yang and Carrier 2001; Yang et al. 2006, 2010; Chen et al. 2018). However, none of the known CIRBP targets are present in our list, indicating alternative ways in which CIRBP can regulate DNA damage. In addition, our list only minimally intersects with transcriptome-wide studies of CIRBP in other biological contexts, suggesting that the functions of CIRBP are context-specific. This is not unusual among RNA binding proteins, which can promote or suppress tumor progression depending on cell type and condition (Pereira et al. 2017). Strikingly, although most known cases of mRNA modulation by CIRBP involve up-regulation of mRNA levels, we find a dominant role of CIRBP in mRNA down-regulation, as most changes after CIRBP depletion include increase in mRNA levels while the opposite is true after CIRBP overexpression. One of the down-regulated targets is CST3, encoding for Cystatin C. Cystatins are inhibitors of cysteine proteases, enzymes which play multiple roles in physiological and pathological settings. CST3 is a major inhibitor of cathepsin B, a matrix protease that promotes invasion and escape from immune recognition by degrading antigens and extracellular matrix components (Keppler 2006). In addition to cathepsin-dependent roles, CST3 can suppress tumorigenesis by binding to the TGF $\beta \|$ receptor and acting as a TGF $\beta$ antagonist (Sokol et al. 2005). Curiously, a recent report found that breast tumors of CST3 knockout mice in a breast cancer model were smaller than their wild-type counterparts, suggesting a tumor-promoting role of CST3 (Završnik et al. 2017). However, in this same model $\mathrm{CST}^{-1-}$ tumors were less aggressive, as they were less capable of metastasizing to the lungs, consistent with a tumor-suppressive role. Our results are consistent with the tumor suppressive capacities of CST3, as CIRBP promotes clonogenicity and anchorage-independent growth by down-regulating CST3 levels. In our model, CST3 seems to act exclusively as a CIRBP mediator, since depletion of CST3 per se has no effect on these traits. Our data are, thus, in agreement with protective roles of CST3 described previously in breast and other cancers such as head and neck, skin, lung, ovarian cancer, and glioma (Konduri et al. 2002; Nishikawa et al. 2004; Strojan et al. 2004; Yu et al. 2010; Mori et al. 2016).

Finally, our network analysis shows that 12 CIRBP targets (S100A11, MAPK11, STK11, CDT1, HOMER3, TRIM28, RPL28, NCAPH2, SF3A2, SLC25A10, SEZ6L2, and MEIS3) in addition to CST3 highly intersect with breast cancer genes. One of them, TRIM28, is known to promote breast cancer metastasis and is an important indicator of disease progression (Wei et al. 2016; Czerwińska et al. 2017; Damineni et al. 2017). However, to our knowledge, no relation with breast cancer has been reported for the remaining $11 \mathrm{CIRBP}$ targets. It will be interesting to analyze the relevance of these targets for breast cancer progression in future studies.

\section{MATERIALS AND METHODS}

\section{Constructs}

The coding sequence of human CIRBP isoform 1 (172 amino acids) was cloned with a carboxy-terminal FLAG-Tag into the MSCV-PIG (PURO-IRES-GFP) retroviral vector using the Gibson Cloning technology (Gibson et al. 2009) with oligos GibF (5'CTAGGCGCCGGAATTAGATCTCTCAGTGGCCGCCATGGCA T3') and GibR (5' TCGTTAACCTCGAGAGATCTTTACTTGTCA TCGTCATCCTTGTAATCCTCGTTGTGTGTAGCGTAAC3'). The construct was verified by sequencing.

\section{Overexpression and depletion}

For CIRBP overexpression, MCF-10A cells were incubated with viral particles containing CIRBP-Flag-MSCV-PIG or empty vector (240.000 cells/2 mL viral suspension/well of a six-well plate), and selected with $1 \mu \mathrm{g} / \mathrm{mL}$ puromycin for $3 \mathrm{~d}$ before collecting cell extracts. The vector contains EGFP, and thereby all cells express this marker. To obtain viral particles, Phoenix A cells were transfected with the respective constructs, selected with $2.5 \mu \mathrm{g} / \mathrm{mL}$ puromycin after $24 \mathrm{~h}$, the supernatant collected $2 \mathrm{~d}$ after puromycin addition and concentrated using Retro- $X$ concentrator (Clontech).

Silencing experiments were performed by transient transfection of siPools (SiTOOLs Biotech) at a concentration of $3 \mathrm{nM}$ using Lipofectamine RNAiMax (Thermo Fisher Scientific) following the manufacturer's protocol. In transfections with two different siPOOLs, $1.5 \mathrm{nM}$ of each were used.

\section{Proliferation, 3D-growth, clonogenicity, and viability assays}

For clonogenicity assays, cells were seeded in triplicates at 2000 cells/well (MCF-7) or 500 cells/well (MCF-10A) in six-well plates, and reverse transfected (i.e., mixed with the transfection mix before plating) with $3 \mathrm{nM}$ siRNA. After $10 \mathrm{~d}$, cells were stained with $0.5 \%$ crystal violet (diluted in $25 \%$ methanol) for $1 \mathrm{~h}$. Plates were scanned and images quantified using FIJI (Guzmán et al. 2014). Cells were then treated with $10 \%$ acetic acid for $30 \mathrm{~min}$ to release the dye, and global crystal violet intensity in the supernatant measured at $\mathrm{OD}_{590}$.

Proliferation assays were performed by seeding cells after reverse transfection of siPOOLs, followed by cell counting every day for 4-5 d in a Neubauer chamber after trypsinization.

For 3D-growth measurements, cells were seeded at 1000 cells/ well in low attachment 96-well plates (Corning) and cultured for 10-14 d with transfection of siPOOLs every $3 \mathrm{~d}$. Cell viability was measured using CellTiter-Glo (Promega) following the recommendations of the vendor.

\section{Cell extracts and western blot}

Cell extracts were prepared by homogenization in RIPA buffer (25 mM Tris-HCL pH 7.6, $150 \mathrm{mM} \mathrm{NaCl}, 1 \%$ NP40, 0.1\% SDS, $1 \%$ Sodium Deoxycholate and $1 \times$ protease inhibitor cocktail from Roche). The cell lysate was spun for $10 \mathrm{~min}$ at $13 \mathrm{Krpm}$ and the supernatant was used or snap-frozen and stored at $-80^{\circ} \mathrm{C}$. The following antibodies were used: anti-CIRBP (Abcam 
TABLE 1. Oligonucleotides used for validation

\begin{tabular}{|c|c|c|}
\hline Gene name & Forward oligonucleotide & Reverse oligonucleotide \\
\hline CDK16 & GCATCCTGTCCAACGAGGAG & GTCGCTATCAAGTCGGGGTG \\
\hline CLDN9 & TTCGACCTTGGCCTGATGAC & CCAGGTGTAGCTTGGGCTTT \\
\hline CST3 & GCAACGACATGTACCACAGC & TTCACCCCAGCTACGATCTGC \\
\hline CYFIP2 & AACGTGGACCTGCTTGAAGA & ACATGATGGAGGAAGGTGGA \\
\hline E2F1 & AGATGGTTATGGTGATCAAAGCC & ATCTGAAAGTTCTCCGAAGAGTCC \\
\hline HRAS & GCTGACCATCCAGCTGATCC & TGCTTCCGGTAGGAATCCTCT \\
\hline LOXL1 & TTTCCCGCTCTCTGATTCTC & TCCTGGAAAAGCTTGGACAT \\
\hline PKM & CGCATGCAGCACCTGATAGC & AGTGACTTGAGGCTCGCAC \\
\hline RALY & GACGACTTCTACGACAGGCTC & CCGGGGTCGCTTCACAG \\
\hline STK11 & AGGCCAACGTGAAGAAGGAAAT & CGTTGTATAACACATCCACCAGC \\
\hline TRIM28 & GATGACAGTGCCACCATTTG & TGACAGTCCAGGTGGAAACA \\
\hline $4 E-B P 1$ & CACCAGCCCTTCCAGTGATG & AACTGTGACTCTTCACCGCC \\
\hline ATR & GGCCAAAGGCAGTTGTATTG & ATGACAGGAGGGAGTTGCTG \\
\hline CCNE1 & GGGAGCTCAAAACTGAAGCA & CCAGCAAATCCAAGCTGTCT \\
\hline HIF1A & CACTACCACTGCCACСACTG & TGGGTAGGAGATGGAGATGC \\
\hline TERT & CGGTGTGCACCAACATCTAC & GGGTTCTTCCAAACTTGCTG \\
\hline TRX & TCAAATGCATGCCAACATTCCA & ATGGTGGCTTCAAGCTTTTCCT \\
\hline ACTB & CCTTTGCCGATCCGCCGC & CCATCACGCCTGGTGCC \\
\hline EIF2A & TGTCACTAACAAGGGACTACTGC & CTGACCAGGATGGACACCAAT \\
\hline
\end{tabular}

ab94999, 1:500), anti-CST3 (Abcam ab109508, dilution 1:1000), anti-Tubulin (Sigma T9026, 1:5000), anti-FLAG (Sigma F3615, 1:5000), anti-SLC25A10 (Sigma HPA023048, 1:500), anti-TRIM28 (Cell Signaling 4124S, 1:500).

\section{RNA-immunoprecipitation (RIP)}

Cells were washed with PBS and resuspended in lysis buffer (100 $\mathrm{mM} \mathrm{KCl}, 5 \mathrm{mM} \mathrm{MgCl}$, $10 \mathrm{mM}$ Hepes pH 7.0, 0.5\% NP40, $1 \mathrm{mM}$ DTT, and RNAsin $40 \mathrm{U} / \mu \mathrm{L}$ ), incubated 4 min on ice, snap-frozen in liquid nitrogen, thawed and sonicated in a Bioruptor Diagenode at high level for $10 \mathrm{~min}$ with cycles of $30 \mathrm{sec}$ ON/OFF at $4^{\circ} \mathrm{C}$. Sonicated cells were spun and the supernatant was precleared with protein A-Dynabeads. On another hand, protein A-Dynabeads were preblocked with $20 \mu \mathrm{g}$ of tRNA in NET buffer (50 mM Tris- $\mathrm{HCl} \mathrm{pH} \mathrm{7.5,} 150 \mathrm{mM} \mathrm{NaCl}, 0.1 \%$ NP40, 1 mM EDTA) for $15 \mathrm{~min}$ at room temperature, washed with NET, and mixed with $8 \mu \mathrm{g}$ of either anti-CIRBP antibody or anti-rabbit IgG for 1 $h$ at $4^{\circ} \mathrm{C}$, washed and resuspended in $100 \mu \mathrm{L}$ of NET. Eighty microlitres of these beads were mixed with $8 \mathrm{mg}$ cell extract, incubated for $1 \mathrm{~h}$ at $4^{\circ} \mathrm{C}$, washed four times with five volumes of NET and resuspended in $100 \mu \mathrm{L}$ of wash buffer. Ten microlitres were used to verify the IP efficiency by western blot. The rest was used for RNA extraction using phenol-chloroform in the presence of $1 \mu \mathrm{L}$ glycoblue (Ambion). DNase treatment was performed with Turbo DNA-free Kit (Ambion). The same steps were used to isolate RNA from the input lysate.

\section{RIP-seq and RNA-seq analysis}

We performed two replicates per RIP-seq or RNA-seq comparison. Library preparation and sequencing were performed at the
CRG Genomics Facility, after assessing RNA quality with Bioanalyzer. The quality of fastq files was assessed with FastQC. Reads were aligned to the human genome (ENSEMBL release 86 for RIP-seq and 88 for RNA-seq) with STAR. The quality of the alignments (BAM files) was assessed with the "rnaseq" module of Qualimap. For RIP-seq, HTSEQ was used to retrieve the number of reads overlapping to each coding gene (option stranded=reverse), first against the whole annotation, and then only against the three last exons of each transcript. The R/Bioconductor package (DESeq2) was used to calculate the pairwise differential expression of genes between experimental groups.

\section{Reverse transcription and quantitative PCR}

Total RNA was isolated using the mirVana kit (Ambion) and treated with DNase using Turbo DNA-free (Ambion). RNA (800 ng) was reversed transcribed using the RevertAid H Minus First Strand cDNA Synthesis Kit (Thermo Fisher) with random hexamers in a final volume of $20 \mu \mathrm{L}$. The resulting cDNA was amplified by Singleplex qPCR using the following Taqman probes: CST3 (4448892-Hs00969174_m1), SLC25A10 (4448892-Hs002017 30_m1), TRIM28 (4448892-Hs00232212_m1). Relative gene expression was calculated by comparative $\Delta \Delta C_{q}$ method according to the MIQE guidelines (Bustin et al. 2009).

For the validation shown in Supplemental Figure S1E, immunoprecipitated RNA (typically $100 \mathrm{ng}$ ) and 1:100 of input were reverse transcribed with Superscript II using oligo(dT) and random hexamers. The resulting cDNA was amplified using the Power Sybr Green PCR master mix (Applied Biosystems) with the oligonucleotides indicated in Table 1. 


\section{SUPPLEMENTAL MATERIAL}

Supplemental material is available for this article.

\section{ACKNOWLEDGMENTS}

We gratefully acknowledge the CRG Bioinformatics Unit and Genomics Facility for high-throughput sequencing and analysis. We thank Cristina Mir, Alex Lyakhovich, Yoelsis García, and Mileydis Pérez for advice and technical help at initial stages of this project. A.I. was supported by a CRG PhD4MD fellowship. F.G. was supported by grants from the Spanish Ministry of Science and Innovation (MICINN, PGC2018-099697-B-I00), "la Caixa" Foundation (ID 100010434) under the agreement LCF/ PR/HR17/52150016, the Catalan Agency for Research and Universities (2017SGR534), and the Centre of Excellence Severo Ochoa. M.E.LL. was supported by grants from the Instituto de Salud Carlos III:PI15/01262 and CP03/00101 cofinanced by the European Regional Fund (ERDF) and AECC Funding ref. GC16173720CARR.

Received May 14, 2020; accepted November 5, 2020.

\section{REFERENCES}

Artero-Castro A, Callejas FB, Castellvi J, Kondoh H, Carnero A, Fernandez-Marcos PJ, Serrano M, Ramón y Cajal S, Lleonart ME. 2009. Cold-inducible RNA-binding protein bypasses replicative senescence in primary cells through extracellular signal-regulated kinase 1 and 2 activation. Mol Cell Biol 29: 1855-1868. doi:10 .1128/MCB.01386-08

Bustin SA, Benes V, Garson JA, Hellemans J, Huggett J, Kubista M, Mueller R, Nolan T, Pfaffl MW, Shipley GL, et al. 2009. The MIQE guidelines: minimum information for publication of quantitative real-time PCR experiments. Clin Chem 55: 611-622. doi:10 .1373/clinchem.2008.112797

Castello A, Fischer B, Hentze MW, Preiss T. 2013. RNA-binding proteins in Mendelian disease. Trends Genet 29: 318-327. doi:10 .1016/j.tig.2013.01.004

Cerami E, Gao J, Dogrusoz U, Gross BE, Sumer SO, Aksoy BA, Jacobsen A, Byrne CJ, Heuer ML, Larsson E, et al. 2012. The cBio cancer genomics portal: an open platform for exploring multidimensional cancer genomics data. Cancer Discov 2: 401-404. doi:10.1158/2159-8290.CD-12-0095

Chang ET, Parekh PR, Yang Q, Nguyen DM, Carrier F. 2016. Heterogenous ribonucleoprotein A18 (hnRNP A18) promotes tumor growth by increasing protein translation of selected transcripts in cancer cells. Oncotarget 7: 10578-10593. doi:10 .18632/oncotarget.7020

Chen J-K, Lin W-L, Chen Z, Liu H. 2018. PARP-1-dependent recruitment of cold-inducible RNA-binding protein promotes doublestrand break repair and genome stability. Proc Natl Acad Sci 115: E1759-E1768. doi:10.1073/pnas. 1713912115

Czerwińska P, Mazurek S, Wiznerowicz M. 2017. The complexity of TRIM28 contribution to cancer. J Biomed Sci 24: 1-14. doi:10 .1186/s12929-017-0374-4

Damineni S, Balaji SA, Shettar A, Nayanala S, Kumar N, Kruthika BS, Subramanian K, Vijayakumar M, Mukherjee G, Gupta V, et al. 2017. Expression of tripartite motif-containing protein 28 in primary breast carcinoma predicts metastasis and is involved in the stemness, chemoresistance, and tumor growth. Tumor Biol 39: 1-16. doi:10.1177/1010428317695919
Gao J, Aksoy BA, Dogrusoz U, Dresdner G, Gross B, Sumer SO, Sun Y, Jacobsen A, Sinha R, Larsson E, et al. 2013. Integrative analysis of complex cancer genomics and clinical profiles using the cBioPortal. Sci Signal 6: pl1. doi:10.1126/scisignal.6273er1

García-Cárdenas JM, Guerrero S, López-Cortés A, ArmendárizCastillo I, Guevara-Ramírez P, Pérez-Villa A, Yumiceba V, Yumiceba V, Zambrano AK, Leone PE, et al. 2019. Post-transcriptional regulation of colorectal cancer: a focus on RNA-binding proteins. Front Mol Biosci 6: 65. doi:10.3389/fmolb.2019.00065

Gerstberger S, Hafner M, Ascano M, Tuschl T. 2014. Evolutionary conservation and expression of human RNA-binding proteins and their role in human genetic disease. In Advances in experimental medicine and biology: systems biology of RNA binding proteins (ed. Yeo GW), pp. 1-32. Springer, New York.

Gibson DG, Young L, Chuang RY, Venter JC, Hutchison CA III, Smith HO. 2009. Enzymatic assembly of DNA molecules up to several hundred kilobases. Nat Methods 6: 343-345. doi:10.1038/ nmeth.1318

Guo X, Wu Y, Hartley RS. 2010. Cold-inducible RNA-binding protein contributes to human antigen R and cyclin E1 deregulation in breast cancer. Mol Carcinog 49: 130-140. doi:10.1002/mc.20582

Guzmán C, Bagga M, Kaur A, Westermarck J, Abankwa D. 2014. ColonyArea: an ImageJ plugin to automatically quantify colony formation in clonogenic assays. PLoS ONE 9: e92444. doi:10 .1371 journal.pone.0092444

Harbeck N, Penault-Llorca F, Cortes J, Gnant M, Houssami N, Poortmans P, Ruddy K, Tsang J, Cardoso F. 2019. Breast cancer. Nat Rev Dis Primers 5: 66. doi:10.1038/s41572-019-0111-2

Hentze MW, Castello A, Schwarzl T, Preiss T. 2018. A brave new world of RNA-binding proteins. Nat Rev Mol Cell Biol 19: 327-341. doi:10.1038/nrm.2017.130

Keppler D. 2006. Towards novel anti-cancer strategies based on cystatin function. Cancer Lett 235: 159-176. doi:10.1016/j.canlet .2005.04.001

Konduri SD, Yanamandra N, Siddique K, Joseph A, Dinh DH, Olivero WC, Gujrati M, Kouraklis G, Swaroop A, Kyritsis AP, et al. 2002. Modulation of cystatin $C$ expression impairs the invasive and tumorigenic potential of human glioblastoma cells. Oncogene 21: 8705-8712. doi:10.1038/sj.onc.1205949

Liu Y, Hu W, Murakawa Y, Yin J, Wang G, Landthaler M, Yan J. 2013. Cold-induced RNA-binding proteins regulate circadian gene expression by controlling alternative polyadenylation. Sci Rep 3: 2054. doi:10.1038/srep02054

Lujan DA, Ochoa JL, Hartley RS. 2018. Cold-inducible RNA binding protein in cancer and inflammation. Wiley Interdiscip Rev RNA 9: 1-10. doi:10.1002/wrna.1462

Masuda T, Itoh K, Higashitsuji $H$, Higashitsuji $H$, Nakazawa N, Sakurai T, Liu Y, Liu Y, Tokuchi H, Fujita T, et al. 2012. Cold-inducible RNA-binding protein (Cirp) interacts with Dyrk1b/Mirk and promotes proliferation of immature male germ cells in mice. Proc Natl Acad Sci 109: 10885-10890. doi:10.1073/pnas .1121524109

Moore S, Järvelin Al, Davis I, Bond GL, Castello A. 2018. Expanding horizons: new roles for non-canonical RNA-binding proteins in cancer. Curr Opin Genet Dev 48: 112-120. doi:10.1016/j.gde .2017 .11 .006

Morf J, Rey G, Schneider K, Stratmann M, Fujita J, Naef F, Schibler U. 2012. Cold-inducible RNA-binding protein modulates circadian gene expression posttranscriptionally. Science 338: 379-383. doi:10.1126/science.1217726

Mori J, Tanikawa C, Funauchi Y, Lo PH, Nakamura Y, Matsuda K. 2016. Cystatin $\mathrm{C}$ as a p53-inducible apoptotic mediator that regulates cathepsin L activity. Cancer Sci 107: 298-306. doi:10.1111/cas .12881 
Nishikawa H, Ozaki Y, Nakanishi T, Blomgren K, Tada T, Arakawa A, Suzumori K. 2004. The role of cathepsin B and cystatin C in the mechanisms of invasion by ovarian cancer. Gynecol Oncol 92: 881-886. doi:10.1016/j.ygyno.2003.11.017

Pereira B, Billaud M, Almeida R. 2017. RNA-binding proteins in cancer: old players and new actors. Trends Cancer 3: 506-528. doi:10.1016/j.trecan.2017.05.003

Qiang X, Yang WL, Wu R, Zhou M, Jacob M, Dong W, Kuncewitch M, Ji Y, Yang H, Wang $H$, et al. 2013. Cold-inducible RNA-binding protein (CIRP) triggers inflammatory responses in hemorrhagic shock and sepsis. Nat Med 19: 1489-1495. doi:10.1038/nm.3368

Sakurai T, Itoh K, Higashitsuji H, Nonoguchi K, Liu Y, Watanabe H, Nakano T, Fukumoto M, Chiba T, Fujita J. 2006. Cirp protects against tumor necrosis factor- $\alpha$-induced apoptosis via activation of extracellular signal-regulated kinase. Biochim Biophys Acta Mol Cell Res 1763: 290-295. doi:10.1016/j.bbamcr.2006.02.007

Sakurai T, Kashida H, Watanabe T, Hagiwara S, Mizushima T, lijima H, Nishida N, Higashitsuji H, Fujita J, Kudo M. 2014. Stress response protein Cirp links inflammation and tumorigenesis in colitis-associated cancer. Cancer Res 74: 6119-6128. doi:10.1158/0008-5472 .CAN-14-0471

Sakurai T, Yada N, Watanabe T, Arizumi T, Hagiwara S, Ueshima K, Nishida N, Fujita J, Kudo M. 2015. Cold-inducible RNA-binding protein promotes the development of liver cancer. Cancer Sci 106: 352-358. doi:10.1111/cas.12611

Sokol JP, Neil JR, Schiemann BJ, Schiemann WP. 2005. The use of cystatin $C$ to inhibit epithelial-mesenchymal transition and morphological transformation stimulated by transforming growth factorß. Breast Cancer Res 7: R844. doi:10.1186/bcr1312

Strojan P, Oblak I, Svetic B, Šmid L, Kos J. 2004. Cysteine proteinase inhibitor cystatin $C$ in squamous cell carcinoma of the head and neck: relation to prognosis. Br J Cancer 90: 1961-1968. doi:10 $.1038 /$ sj.bjc. 6601830
Wei C, Cheng J, Zhou B, Zhu L, Khan Md A, He T, Zhou S, He J, Lu X, $\mathrm{Chen} \mathrm{H}$, et al. 2016. Tripartite motif containing 28 (TRIM28) promotes breast cancer metastasis by stabilizing TWIST1 protein. Sci Rep 6: 1-12. doi:10.1038/s41598-016-0001-8

Wurth L, Gebauer F. 2015. RNA-binding proteins, multifaceted translational regulators in cancer. Biochim Biophys Acta Gene Regul Mech 1849: 881-886. doi:10.1016/j.bbagrm.2014.10.001

Yang C, Carrier F. 2001. The UV-inducible RNA-binding protein A18 (A18 hnRNP) plays a protective role in the genotoxic stress response. J Biol Chem 276: 47277-47284. doi:10.1074/jbc .M105396200

Yang R, Weber DJ, Carrier F. 2006. Post-transcriptional regulation of thioredoxin by the stress inducible heterogenous ribonucleoprotein A18. Nucleic Acids Res 34: 1224-1236. doi:10.1093/nar/ gkj519

Yang R, Zhan M, Rao Nalabothula N, Yang Q, Indig FE, Carrier F. 2010. Functional significance for a heterogenous ribonucleoprotein A18 signature RNA motif in the $3^{\prime}$-untranslated region of ataxia telangiectasia mutated and rad3-related (ATR) transcript. J Biol Chem 285: 8887-8893. doi:10.1074/jbc.M109.013128

Yu W, Liu J, Shi MA, Wang J, Xiang M, Kitamoto S, Wang B, Sukhova GK, Murphy GF, Orasanu G, et al. 2010. Cystatin C deficiency promotes epidermal dysplasia in K14-HPV16 transgenic mice. PLoS One 5: e13973. doi:10.1371/journal.pone.0013973

Završnik J, Butinar M, Prebanda MT, Aleksander K, Vidmar R, Fonovic M, Grubb A, Turk V, Turk B, Vasiljeva O. 2017. Cystatin $C$ deficiency suppresses tumor growth in a breast cancer model through decreased proliferation of tumor cells. Oncotarget 8: 73793-73809. doi:10.18632/oncotarget.17379

Zhang Y, Wu Y, Mao P, Li F, Han X, Zhang Y, Jiang S, Chen Y, Huang J, Liu D, et al. 2016. Cold-inducible RNA-binding protein CIRP/ hnRNP A18 regulates telomerase activity in a temperature-dependent manner. Nucleic Acids Res 44: 761-775. doi:10.1093/nar/ gkv1465 

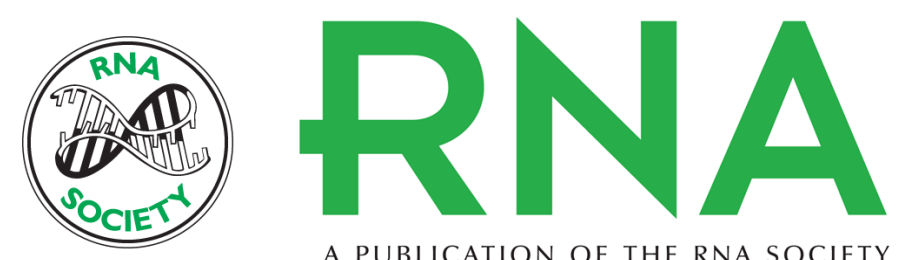

A PUBLICATION OF THE RNA SOCIETY

\section{Cold-inducible RNA binding protein promotes breast cancer cell malignancy by regulating Cystatin C levels}

Alberto Indacochea, Santiago Guerrero, Macarena Ureña, et al.

RNA 2021 27: 190-201 originally published online November 10, 2020

Access the most recent version at doi:10.1261/rna.076422.120

\section{Supplemental http://rnajournal.cshlp.org/content/suppl/2020/11/10/rna.076422.120.DC1 Material}

References This article cites 39 articles, 9 of which can be accessed free at: http://rnajournal.cshlp.org/content/27/2/190.full.html\#ref-list-1

Open Access Freely available online through the RNA Open Access option.

Creative This article, published in RNA, is available under a Creative Commons License Commons (Attribution 4.0 International), as described at

License http://creativecommons.org/licenses/by/4.0/.

Email Alerting Receive free email alerts when new articles cite this article - sign up in the box at the Service top right corner of the article or click here.

\section{|||||||| Providing Precise Solutions for} your research.

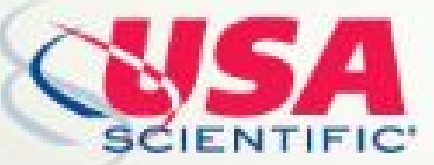

To subscribe to $R N A$ go to:

http://rnajournal.cshlp.org/subscriptions

(C) 2021 Indacochea et al.; Published by Cold Spring Harbor Laboratory Press for the RNA Society 